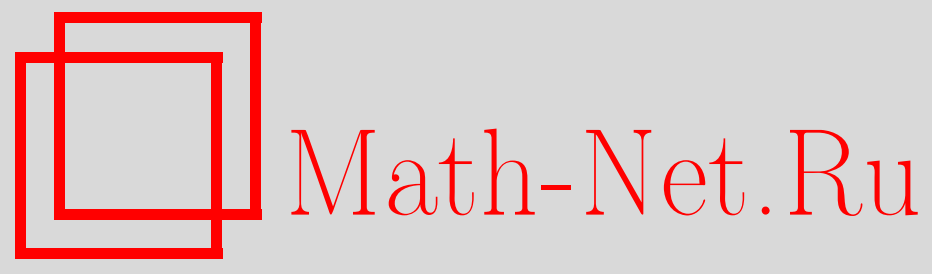

Р. Дуади, М. Йор, А. Н. Ширяев, О вероятностных характеристиках величин "падения" в стандартном броуновском движении, Теория вероятн. и ее примен., 1999, том 44, выпуск 1, 3-13

DOI: https://doi.org/10.4213/tvp594

Использование Общероссийского математического портала MathNet.Ru подразумевает, что вы прочитали и согласны с пользовательским соглашением

http: //www . mathnet.ru/rus/agreement

Параметры загрузки:

IP : 3.85 .5 .30

26 апреля 2023 г., 16:28:54

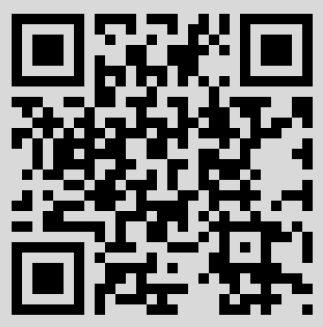


(C) 1999 г. ДУАДИ Р., ЙОР М., ШИРЯЕВ А. Н.**

\section{О ВЕРОЯТНОСТНЫХ ХАРАКТЕРИСТИКАХ ВЕЛИЧИН «ПАДЕНИЯ» В СТАНДАРТНОМ БРОУНОВСКОМ ДВИЖЕНИИ}

Для броуновского движения $B=\left(B_{t}\right)_{t \leqslant 1}$ с $B_{0}=0, \mathbf{E} B_{t}=0$, $\mathrm{E} B_{t}^{2}=t$ рассматриваются вопросы о распределениях вероятностей и их характеристиках для величин

$$
\begin{aligned}
& \mathbb{D}=\sup _{0 \leqslant t \leqslant t^{\prime} \leqslant 1}\left(B_{t}-B_{t^{\prime}}\right), \quad \mathbb{D}_{1}=B_{\sigma}-\inf _{\sigma \leqslant t^{\prime} \leqslant 1} B_{t^{\prime}} \\
& \mathbb{D}_{2}=\sup _{0 \leqslant t \leqslant \sigma^{\prime}} B_{t}-B_{\sigma^{\prime}}
\end{aligned}
$$

где $\sigma$ и $\sigma^{\prime}$ - моменты (немарковские) абсолютного максимума и абсолютного минимума броуновского движения на $[0,1]$ (т.е. $B_{\sigma}=$ $\left.\sup _{0 \leqslant t \leqslant 1} B_{t}, B_{\sigma^{\prime}}=\inf _{0 \leqslant t^{\prime} \leqslant 1} B_{t^{\prime}}\right)$.

Ключевые слова и фразы: броуновское движение, величины «падения»и «размаха», теорема Леви, броуновский меандр.

\section{§ 1. Введение. Формулировка результатов}

1. На вероятностном пространстве $(\Omega, \mathscr{F}, \mathbb{P})$ рассматривается станцартное броуновское движение $B=\left(B_{t}\right)_{0 \leqslant t \leqslant 1}$ (с $B_{0}=0, \mathbb{E} B_{t}=0$, $\left.\mathbf{E} B_{t}^{2}=t\right)$.

Наш основной интерес будет связан с изучением вероятностных свойств величин

$$
\begin{aligned}
& \mathbb{D}=\sup _{0 \leqslant t \leqslant t^{\prime} \leqslant 1}\left(B_{t}-B_{t^{\prime}}\right) \\
& \mathbb{D}_{1}=B_{\sigma}-\inf _{\sigma \leqslant t^{\prime} \leqslant 1} B_{t^{\prime}}, \\
& \mathbb{D}_{2}=\sup _{0 \leqslant t \leqslant \sigma^{\prime}} B_{t}-B_{\sigma^{\prime}}
\end{aligned}
$$

${ }^{*}$ Laboratoire de Probabilités, Université Paris VI, 4 Place Jussieu, Tour 56, F-75252 Paris Cédex 05, France.

${ }^{* *}$ Математический институт им. В. А. Стеклова РАН, ул. Губкина, 8, 117966 Москва, ГСП-1, Россия. 
где $\sigma$ и $\sigma^{\prime}$ определяются (P-п.н. однозначно) соотношениями

$$
\begin{aligned}
B_{\sigma} & =\sup _{0 \leqslant t \leqslant 1} B_{t} \\
B_{\sigma^{\prime}} & =\inf _{0 \leqslant t^{\prime} \leqslant 1} B_{t^{\prime}} .
\end{aligned}
$$

Из приведенных определений ясно, что величина $\mathbb{D}$ характеризует максимально возможное «падение» траекторий броуновского движения на временном интервале $[0,1]$.

Величина $\mathbb{D}_{1}$ показывает, насколько может «падать» траектория броуновского движения от абсолютного (на $[0,1])$ максимума $B_{\sigma}$ к (час-

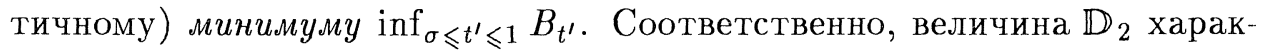
теризует «падение» от (частичного) максимума $\sup _{0 \leqslant t \leqslant \sigma^{\prime}} B_{t}$ к абсолютному (на $[0,1])$ минимуму $B_{\sigma^{\prime}}$.

Полезно отметить, что моменты $\sigma$ и $\sigma^{\prime}$ являются немарковскими.

2. Понятно, что

$$
\mathbb{D}=\left\{\begin{array}{l}
\sup _{0 \leqslant t \leqslant 1}\left(B_{t}-\inf _{t \leqslant t^{\prime} \leqslant 1} B_{t^{\prime}}\right) \geqslant \mathbb{D}_{1}, \\
\sup _{0 \leqslant t^{\prime} \leqslant 1}\left(\sup _{0 \leqslant t \leqslant t^{\prime}} B_{t}-B_{t^{\prime}}\right) \geqslant \mathbb{D}_{2} .
\end{array}\right.
$$

Тем самым,

$$
\max \left(\mathbb{D}_{1}, \mathbb{D}_{2}\right) \leqslant \mathbb{D}
$$

(Можно убедиться в том, что в (6) на самом деле имеет место знак равенства: $\max \left(\mathbb{D}_{1}, \mathbb{D}_{2}\right)=\mathbb{D}$.)

Если обозначить

$$
\mathbb{R}=\sup _{0 \leqslant t \leqslant 1} B_{t}-\inf _{0 \leqslant t \leqslant 1} B_{t}
$$

— «размах» броуновского движения, то найдем, что

$$
\mathbb{R}=\sup _{0 \leqslant t, t^{\prime} \leqslant 1}\left(B_{t}-B_{t^{\prime}}\right)
$$

и

$$
\max \left(\mathbb{D}_{1}, \mathbb{D}_{2}\right)=\mathbb{D} \leqslant \mathbb{R}
$$

Хорошо известно, что рассмотрение статистики $\mathbb{R}$ полезно при апостериорном анализе поведения траекторий процесса $B$ на временном интервале $[0,1]$. В то же время, статистики $\mathbb{D}, \mathbb{D}_{1}, \mathbb{D}_{2}$ дают больше информации о текущем поведении траекторий, что особенно важно тогда, когда надо учитывать временную упорядоченность производимых наблюдений. 
3. Переходя к формулировкам результатов относительно вероятностных распределений и их характеристик статистик $\mathbb{D}, \mathbb{D}_{1}$ и $\mathbb{D}_{2}$, прежде всего заметим, что по распределению величины $\mathbb{D}_{1}$ и $\mathbb{D}_{2}$ совпадают:

$$
\mathbb{D}_{1} \stackrel{\text { law }}{=} \mathbb{D}_{2} \text {. }
$$

(Это легко следует из того, что процесс $\widehat{B}=\left(\widehat{B}_{t}\right)_{0 \leqslant t \leqslant 1}$ с $\widehat{B}_{t}=B_{1}-B_{1-t}$ также является броуновским движением.)

Теорема 1. Для стандартного броуновского движения

$$
\mathbb{D} \stackrel{\text { law }}{=} \sup _{0 \leqslant t \leqslant 1}\left|B_{t}\right|
$$

Среднее значение

$$
\mathbf{E} \mathbb{D}=\sqrt{\frac{\pi}{2}} \quad(=1.2533 \ldots)
$$

и функция распределения $F_{\mathbb{D}}(x)=\mathbf{P}(\mathbb{D} \leqslant x)$ задается формулой

$$
F_{\mathbb{D}}(x)=1-\frac{1}{\sqrt{2 \pi}} \sum_{k=-\infty}^{\infty} \int_{-x}^{x}\left[e^{-(y+4 k x)^{2} / 2}-e^{-(y+2 x+4 k x)^{2} / 2}\right] d y .
$$

Теорема 2. Для стандартного броуновского движения

$$
\mathbb{D}_{1} \stackrel{\text { law }}{=} \sup _{g \leqslant t \leqslant 1}\left|B_{t}\right|
$$

$2 \partial e$

$$
g=\sup \left\{t \leqslant 1: B_{t}=0\right\}
$$

- момент последнего обращения в нуль прочесса $B=\left(B_{t}\right)_{0 \leqslant t \leqslant 1}$.

Среднее значение

$$
\mathbf{E D}_{1}=\sqrt{\frac{8}{\pi}} \ln 2 \quad(=1.1061 \ldots)
$$

и функция распределения $F_{\mathbb{D}_{1}}(x)=\mathbf{P}\left(\mathbb{D}_{1} \leqslant x\right)$ имеет плотность (рис. 1$)$

$$
f_{\mathbb{D}_{1}}(x)=\sqrt{\frac{8}{\pi}} \sum_{k=1}^{\infty}(-1)^{k-1} k e^{-k^{2} x^{2} / 2}, \quad x>0 .
$$

4. Ряд (16) весьма схож с теми рядами, которыми описываются распределение вероятностей $F_{\mathbb{K}}(x)$ в критерии согласия Колмогорова и распределение вероятностей $F_{\mathbb{R}}(x)$ статистики «размаха» $\mathbb{R}$.

Именно, функция распределения

$$
F_{\mathbb{K}}(x)=\mathbf{P}\left(\operatorname{sip}_{0 \leqslant t \leqslant 1}|b(t)| \leqslant x\right)
$$


где $b=(b(t))_{0 \leqslant t \leqslant 1}-$ броуновский мост $\left((b(t) ; t \leqslant 1) \stackrel{\text { law }}{=}\left(B_{t}-t B_{1} ; t \leqslant 1\right)\right)$, задается при $x>0$ формулой $([7],[5],[4])$

$$
F_{\mathbb{K}}(x)=1-2 \sum_{k=1}^{\infty}(-1)^{k-1} e^{-2 k^{2} x^{2}}
$$

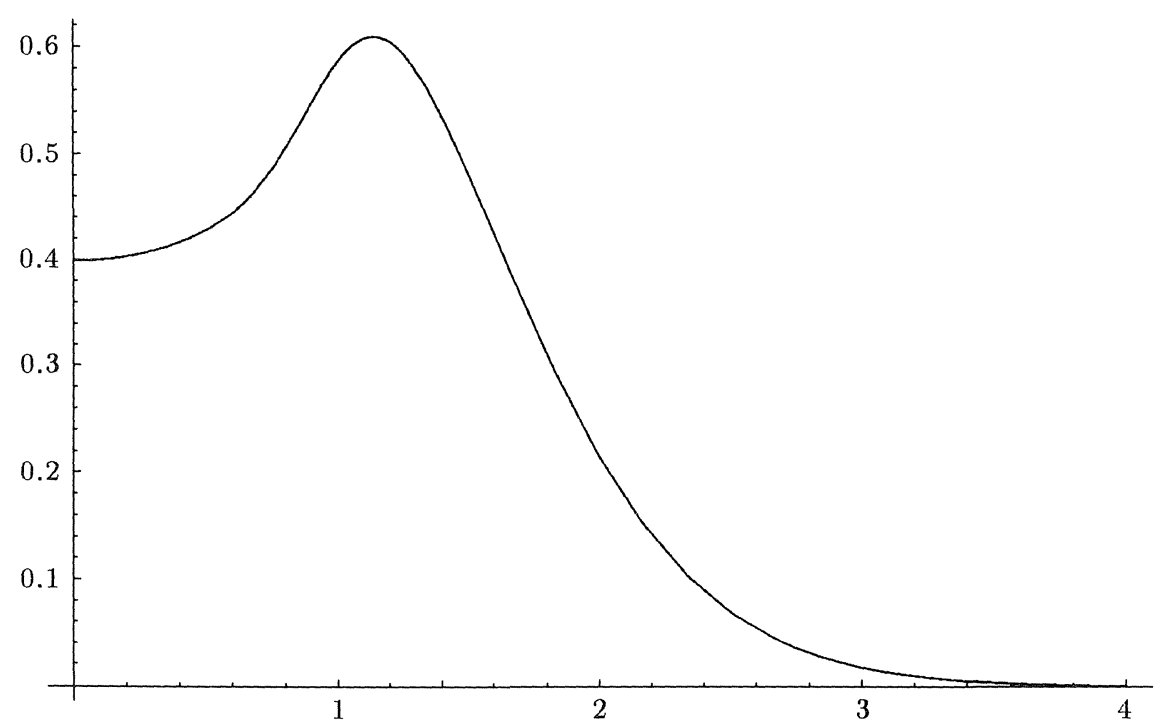

Рис. 1. График функции $f_{\mathbb{D}_{1}}(x)=\sqrt{8 / \pi} \sum_{k=1}^{\infty}(-1)^{k-1} k e^{-k^{2} x^{2} / 2}, x>0$.

или, равносильно, формулой

$$
F_{\mathbb{K}}(x)=\frac{\sqrt{2 \pi}}{x} \sum_{k=1}^{\infty} e^{-(2 k-1)^{2} \pi^{2} / x^{2}} .
$$

Известно также (см., например, [6]), что распределение вероятностей $\mathbb{F}_{\mathbb{R}}(x)=\mathbb{P}(\mathbb{R} \leqslant x)$ имеет плотность (рис. 2 )

$$
f_{\mathbb{R}}(x)=\frac{8}{\sqrt{2 \pi}} \sum_{k=1}^{\infty}(-1)^{k-1} k^{2} e^{-k^{2} x^{2} / 2}, \quad x>0 .
$$

Из (18) и (19) видим, что плотность $f_{\mathbb{K}}(x)=F_{\mathbb{K}}^{\prime}(x)$ определяется формулой (рис. 3)

$$
f_{\mathbb{K}}(x)=8 x \sum_{k=1}^{\infty}(-1)^{k-1} k^{2} e^{-2 k^{2} x^{2}}, \quad x>0,
$$

и при этом

$$
f_{\mathbb{R}}(x)=\sqrt{\frac{2}{\pi}} \frac{1}{x} f_{\mathbb{K}}\left(\frac{x}{2}\right)
$$


Отсюда, в частности, заключаем, что

$$
\mathbf{E} \mathbb{R}=\sqrt{\frac{8}{\pi}} \quad(=1.5957 \ldots)
$$

(cp. c (8), (11), (15)).

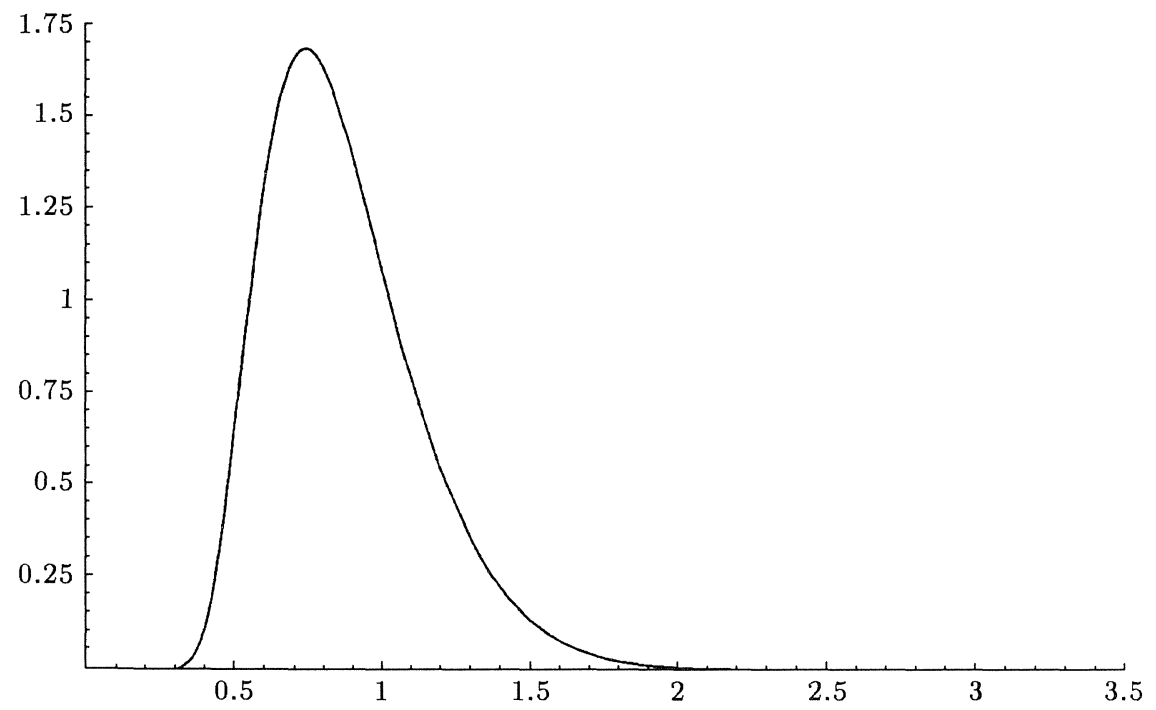

Рис. 2. График функции $f_{\mathbb{R}}(x)=8 / \sqrt{2 \pi} \sum_{k=1}^{\infty}(-1)^{k-1} k^{2} e^{-k^{2} x^{2} / 2}, x>0$.

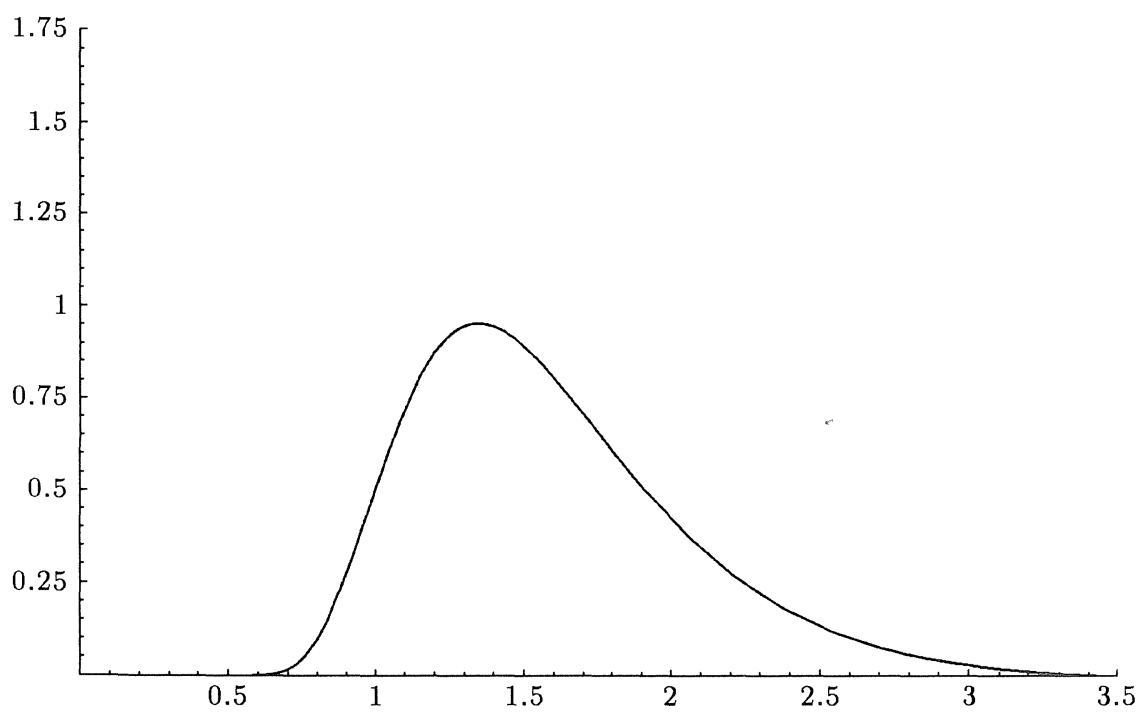

Рис. 3. График функции $f_{\mathbb{K}}(x)=8 \sum_{k=1}^{\infty}(-1)^{k-1} k^{2} x e^{-2 k^{2} x^{2}}, x>0$. 


\section{§ 2. Доказательство теоремы 1}

1. Если установлено, что $\mathbb{D} \stackrel{\text { law }}{=} \sup _{0 \leqslant t \leqslant 1}\left|B_{t}\right|$ (свойство (10)), то формула (12) будет следовать из известных результатов относительно распределения $\mathbb{P}\left(\sup _{0 \leqslant t \leqslant 1}\left|B_{t}\right| \leqslant x\right)$. (См., например, $[1$, с. 250 , ф-ла 1.1.4].)

Доказательство же требуемого свойства (10) весьма просто. Обозначим

$$
M_{t}=\sup _{0 \leqslant u \leqslant t} B_{u}
$$

и $L_{t}-$ локальное время броуновского движения $B$ на $[0, t]$ :

$$
L_{t}=\lim _{\varepsilon \downarrow 0} \frac{1}{2 \varepsilon} \int_{0}^{t} I\left(\left|B_{s}\right| \leqslant \varepsilon\right) d s .
$$

Согласно одному из фундаментальных свойств броуновского движения,

$$
\left(M_{t}-B_{t}, M_{t} ; t \leqslant 1\right) \stackrel{\text { law }}{=}\left(\left|B_{t}\right|, L_{t} ; t \leqslant 1\right)
$$

(«теорема П. Леви» $[9$, с. 230]).

Поэтому

$$
\begin{aligned}
\mathbb{D} & =\sup _{0 \leqslant t \leqslant t^{\prime} \leqslant 1}\left(B_{t}-B_{t^{\prime}}\right) \\
& =\sup _{0 \leqslant t^{\prime} \leqslant 1}\left(\sup _{0 \leqslant t \leqslant t^{\prime}} B_{t}-B_{t^{\prime}}\right)=\sup _{0 \leqslant t^{\prime} \leqslant 1}\left(M_{t^{\prime}}-B_{t^{\prime}}\right) \stackrel{\text { law }}{=} \sup _{0 \leqslant t \leqslant 1}\left|B_{t}\right| .
\end{aligned}
$$

2. Формула $\mathbb{E} \mathbb{D}=\sqrt{\pi / 2}$ известна, видимо, многим и может быть получена из (12).

Для полноты изложения приведем два ее вывода, не апеллирующие непосредственно к формуле (12) для распределения $F_{\mathbb{D}}(x)=\mathbf{P}(\mathbb{D} \leqslant x)$.

Пусть $\beta=\left(\beta_{t}\right)_{t \geqslant 0}-$ броуновское движение, $S_{1}=\inf \left\{t \geqslant 0:\left|\beta_{t}\right|=1\right\}$. Если воспользоваться свойством автомодельности: для $a>0$

$$
\left(\beta_{a t} ; t \geqslant 0\right) \stackrel{\text { law }}{=}\left(a^{1 / 2} \beta_{t} ; t \geqslant 0\right),
$$

то найдем, что для $x>0$

$$
\begin{aligned}
\mathbf{P}\left(\sup _{0 \leqslant t \leqslant 1}\left|\beta_{t}\right| \leqslant x\right) & =\mathbf{P}\left(\sup _{0 \leqslant t \leqslant 1}\left|\beta_{t / x^{2}}\right| \leqslant 1\right)=\mathbf{P}\left(\sup _{0 \leqslant t \leqslant 1 / x^{2}}\left|\beta_{t}\right| \leqslant 1\right) \\
& =\mathbb{P}\left(S_{1} \geqslant \frac{1}{x^{2}}\right)=\mathbf{P}\left(\frac{1}{\sqrt{S_{1}}} \leqslant x\right) .
\end{aligned}
$$

Иначе говоря,

$$
\sup _{0 \leqslant t \leqslant 1}\left|\beta_{t}\right| \stackrel{\text { law }}{=} \frac{1}{\sqrt{S_{1}}}
$$


Из свойств нормального распределения следует, что для $\sigma>0$

$$
\sqrt{\frac{2}{\pi}} \int_{0}^{\infty} e^{-x^{2} /\left(2 \sigma^{2}\right)} d x=\sigma
$$

Тем самым,

$$
\mathbf{E D}=\mathbf{E} \sup _{0 \leqslant t \leqslant 1}\left|\beta_{t}\right|=\mathbf{E} \frac{1}{\sqrt{S_{1}}}=\sqrt{\frac{2}{\pi}} \int_{0}^{\infty} \mathbf{E} e^{-x^{2} S_{1} / 2} d x
$$

Для величины $S_{1}$ хорошо известно (см., например, [9, с. 68], [10, с. 303]) преобразование Лапласа

$$
\mathbf{E} e^{-\lambda S_{1}}=\frac{1}{\operatorname{ch} \sqrt{2 \lambda}}
$$

Поэтому

$$
\begin{aligned}
\mathbf{E D} & =\sqrt{\frac{2}{\pi}} \int_{0}^{\infty} \frac{d x}{\operatorname{ch} x}=2 \sqrt{\frac{2}{\pi}} \int_{0}^{\infty} \frac{e^{x} d x}{e^{2 x}+1}=2 \sqrt{\frac{2}{\pi}} \int_{1}^{\infty} \frac{d y}{1+y^{2}} \\
& =\left.2 \sqrt{\frac{2}{\pi}} \operatorname{arctg} x\right|_{1} ^{\infty}=2 \sqrt{\frac{2}{\pi}} \frac{\pi}{4}=\sqrt{\frac{\pi}{2}}
\end{aligned}
$$

Второй вывод формулы $\mathbf{E D}=\sqrt{\pi / 2}$ основан на том свойстве, что

$$
\sup _{0 \leqslant t \leqslant 1}\left|B_{t}\right| \stackrel{\text { law }}{=} \frac{1}{2} \int_{0}^{1} \frac{d s}{R_{s}^{(2)}}
$$

$($ см. $[9$, с. 250$])$, где $\left(R_{s}^{(2)}\right)_{s \leqslant 1}$ - процесс Бесселя порядка 2 , для которого

$$
R_{s}^{(2)}=\widehat{\beta}_{s}+\frac{1}{2} \int_{0}^{s} \frac{d u}{R_{u}^{(2)}}
$$

где $\widehat{\beta}=\left(\widehat{\beta}_{s}\right)_{s \leqslant 1}$ - некоторое броуновское движение.

Из (21) и (22) непосредственно видим, что

$$
\mathbf{E D}=\mathbf{E} \sup _{0 \leqslant t \leqslant 1}\left|\beta_{t}\right|=\mathbf{E} R_{1}^{(2)}=\mathbf{E} \sqrt{\xi_{1}^{2}+\xi_{2}^{2}},
$$

где $\xi_{1}$ и $\xi_{2}$ - независимые стандартные гауссовские величины с параметрами 0 и 1 . Поскольку $\mathbf{E} \sqrt{\xi_{1}^{2}+\xi_{2}^{2}}=\sqrt{\pi / 2}$, то снова приходим к требуемой формуле $\mathbf{E D}=\sqrt{\pi / 2}$. 


\section{§ 3. Доказательство теоремы 2}

1. Из теоремы Леви (20) следует, что

$$
\left(M_{t}-B_{t}, M_{t}, B_{t} ; t \leqslant 1\right) \stackrel{\text { law }}{=}\left(\left|B_{t}\right|, L_{t}, L_{t}-\left|B_{t}\right| ; t \leqslant 1\right) .
$$

Отсюда можно заключить, что

$$
\left(M_{t}-B_{t}, M_{t}, B_{t} ; \sigma \leqslant t \leqslant 1\right) \stackrel{\text { law }}{=}\left(\left|B_{t}\right|, L_{t}, L_{t}-\left|B_{t}\right| ; g \leqslant t \leqslant 1\right) .
$$

Значит,

$$
\begin{aligned}
\left(B_{\sigma}, \sup _{\sigma \leqslant t \leqslant 1}\left(M_{t}-B_{t}-M_{t}\right)\right) & \stackrel{\text { law }}{=}\left(L_{g}-\left|B_{g}\right|, \sup _{g \leqslant t \leqslant 1}\left(\left|B_{t}\right|-L_{t}\right)\right), \\
& =\left(L_{g}, \sup _{g \leqslant t \leqslant 1}\left|B_{t}\right|-L_{g}\right)
\end{aligned}
$$

поскольку $B_{g}=0$ и $L_{t}=L_{g}$ для $g \leqslant t \leqslant 1$ в силу (14).

Поэтому

$$
\begin{aligned}
\mathbb{D}_{1} & =B_{\sigma}-\inf _{\sigma \leqslant t \leqslant 1} B_{t}=B_{\sigma}+\sup _{\sigma \leqslant t \leqslant 1}\left(-B_{t}\right) \\
& =B_{\sigma}+\sup _{\sigma \leqslant t \leqslant 1}\left(M_{t}-B_{t}-M_{t}\right) \stackrel{\text { law }}{=} L_{g}+\sup _{g \leqslant t \leqslant 1}\left|B_{t}\right|-L_{g}=\sup _{g \leqslant t \leqslant 1}\left|B_{t}\right|,
\end{aligned}
$$

что доказывает утверждение (13).

2. Обратимся к доказательству формулы (16). Пусть $m=$ $\left(m_{u}\right)_{0 \leqslant u \leqslant 1}$ - стандартный броуновский меандр (meander), или «броуновская извилина» $([9$, с. 468$],[11 ; 12.3 .2],[2])$ :

$$
m_{u} \equiv \frac{1}{\sqrt{1-g}}\left|B_{g+u(1-g)}\right| \text {. }
$$

Вместе с (13) отсюда заключаем, что

$$
\mathbb{D}_{1} \stackrel{\text { law }}{=} \sqrt{1-g} \sup _{0 \leqslant u \leqslant 1} m_{u} .
$$

Для отыскания распределения величины $\sqrt{1-g} \sup _{0 \leqslant u \leqslant 1} m_{u}$ заметим, прежде всего, что $g \stackrel{\text { law }}{=} 1-g$, причем (согласно «первому закону арксинуса», $[9$, с. 106]) плотность

$$
f_{g}(x)=\frac{1}{\pi \sqrt{x(1-x)}}, \quad 0<x<1 .
$$

Известно также, что $g$ и $\sup _{0 \leqslant u \leqslant 1} m_{u}$ независимы (см. $[9$, с. 468]).

Далее, если $\varepsilon \sim \exp \left(\frac{1}{2}\right)$, т.е. $\varepsilon$ является экспоненциально распределенной случайной величиной с параметром $\frac{1}{2}$, и не зависит от броуновского движения $B$, то непосредственно проверяется, что

$$
\varepsilon g \stackrel{\text { law }}{=} N^{2}
$$


где $N \sim \mathscr{N}(0,1)$, т.е. $N$ имеет стандартное гауссовское распределение с параметрами 0 и 1.

Далее, известно (см. [9, с. 484], [2, с. 3 и 6]), что

$$
\mathbf{P}\left(|N| \sup _{0 \leqslant u \leqslant 1} m_{u} \leqslant x\right)=\operatorname{th} \frac{x}{2} \text {. }
$$

Следовательно,

$$
\mathbf{P}\left(\sqrt{\varepsilon} \mathbb{D}_{1} \leqslant x\right)=\mathbf{P}\left(\sqrt{\varepsilon g} \sup _{0 \leqslant u \leqslant 1} m_{u} \leqslant x\right)=\text { th } \frac{x}{2} .
$$

Положим

$$
\eta=\frac{1}{\mathbb{D}_{1}}, \quad f_{\eta}(y)=\frac{d}{d y} \mathbf{P}(\eta \leqslant y) .
$$

Тогда в силу независимости $\varepsilon$ и $\eta$

$$
\mathbf{P}\left(\varepsilon \leqslant x^{2} \eta \mid \eta=y\right)=\mathbf{P}\left(\varepsilon \leqslant x^{2} y\right)=1-e^{-x^{2} y / 2} .
$$

Поэтому согласно (26)

$$
1-\int_{0}^{\infty} e^{-x^{2} y / 2} f_{\eta}(y) d y=\operatorname{th} \frac{x}{2}
$$

или, равносильно,

$$
\begin{aligned}
\int_{0}^{\infty} e^{-x^{2} y / 2} f_{\eta}(y) d y & =1-\operatorname{th} \frac{x}{2}=\frac{2 e^{-x}}{1+e^{-x}} \\
& =2 \sum_{k=0}^{\infty}(-1)^{k} e^{-(k+1) x} .
\end{aligned}
$$

Пусть $T_{a}=\inf \left\{t \geqslant 0: \beta_{t}=a\right\}$, где $\beta=\left(\beta_{t}\right)_{t \geqslant 0}-$ броуновское движение. Известно (см., например, $[9$, с. 68], $[10$, с. 302,303$])$, что распределение вероятностей $F_{T_{a}}(t)=\mathrm{P}\left(T_{a} \leqslant t\right), a>0$, имеет плотность

$$
f_{T_{a}}(t)=\frac{a}{\sqrt{2 \pi t^{3}}} e^{-a^{2} /(2 t)}, \quad t>0
$$

и преобразование Лапласа $\mathbf{E} e^{-\lambda T_{a}}=e^{-a \sqrt{2 \lambda}}, \lambda>0$. Поэтому, беря $\lambda=$ $x^{2} / 2$, находим, что для $x \geqslant 0$

$$
e^{-a x}=\int_{0}^{\infty} \frac{a}{\sqrt{2 \pi t^{3}}} e^{-a^{2} /(2 t)} e^{-x^{2} t / 2} d t
$$

Отсюда и из (27) получаем

$$
\begin{aligned}
\int_{0}^{\infty} e^{-x^{2} y / 2} f_{\eta}(y) d y & =2 \sum_{k=0}^{\infty}(-1)^{k} e^{-(k+1) x} \\
& =2 \int_{0}^{\infty} e^{-x^{2} y / 2} \sum_{k=0}^{\infty}(-1)^{k} \frac{k+1}{\sqrt{2 \pi y^{3}}} e^{-(k+1)^{2} /(2 y)} d y
\end{aligned}
$$


и, значит, в силу произвольности $x>0$,

$$
f_{\eta}(y)=2 \sum_{k=0}^{\infty}(-1)^{k} \frac{k+1}{\sqrt{2 \pi y^{3}}} e^{-(k+1)^{2} /(2 y)}, \quad y>0 .
$$

Наконец, для $x>0$

$$
F_{\mathbb{D}_{1}}(x)=\mathbf{P}\left(\mathbb{D}_{1} \leqslant x\right)=\mathbf{P}\left(\eta \geqslant \frac{1}{x^{2}}\right)=1-\mathbf{P}\left(\eta<\frac{1}{x^{2}}\right)
$$

и поэтому

$$
\begin{aligned}
f_{\mathbb{D}_{1}}(x) & =\frac{2}{x^{3}} f_{\eta}\left(\frac{1}{x^{2}}\right)=\frac{4}{\sqrt{2 \pi}} \sum_{k=0}^{\infty}(-1)^{k}(k+1) e^{-(k+1)^{2} x^{2} / 2} \\
& =\sqrt{\frac{8}{\pi}} \sum_{k=1}^{\infty}(-1)^{k-1} k e^{-k^{2} x^{2} / 2},
\end{aligned}
$$

что и доказывает формулу (16).

3. Формулу $\mathbf{E D}_{1}=\sqrt{8 / \pi} \ln 2$ можно получить из (16):

$$
\begin{aligned}
\mathbf{E D}_{1} & =\int_{0}^{\infty} x f_{\mathbb{D}_{1}}(x) d x=\sqrt{\frac{8}{\pi}} \int_{0}^{\infty}\left(\sum_{k=1}^{\infty}(-1)^{k-1} k x e^{-k^{2} x^{2} / 2}\right) d x \\
& =\sqrt{\frac{8}{\pi}} \sum_{k=1}^{\infty}(-1)^{k-1} \int_{0}^{\infty} k x e^{-k^{2} x^{2} / 2} d x \\
& =\sqrt{\frac{8}{\pi}} \sum_{k=1}^{\infty}(-1)^{k-1} \frac{1}{k}=\sqrt{\frac{8}{\pi}} \ln 2 .
\end{aligned}
$$

(В данном выводе необходимо, конечно, обосновывать возможность смены порядка интегрирования и суммирования. Подобная смена не вызывает трудностей, если вместо $\mathbf{E D}_{1}$ оперировать с $\mathbf{E D}_{1} I\left(a<\mathbb{D}_{1}<A\right)$, где $0<a<A<\infty$. Затем следует воспользоваться тем, что $\mathbf{E D}_{1} I(a<$ $\left.\mathbb{D}_{1}<A\right) \uparrow \mathbf{E} \mathbb{D}_{1}$ при $a \downarrow 0, A \uparrow \infty$.)

Требуемую формулу $\mathbf{E D}_{1}=\sqrt{8 / \pi} \ln 2$ можно получить также из следующих соображений.

Из (23)

$$
\mathbf{E} \mathbb{D}_{1}=\mathbf{E} \sqrt{1-g} S_{m}=\mathbf{E} \sqrt{1-g} \mathbf{E} S_{m}
$$

где $S_{m}=\sup _{0 \leqslant u \leqslant 1} m_{u}$ и второе равенство следует из уже отмечавшегося выше свойства независимости величин $g$ и $S_{m}$.

В силу (24)

$$
\mathrm{E} \sqrt{1-g}=\frac{1}{\pi} \int_{0}^{1} \frac{d x}{\sqrt{x}}=\frac{2}{\pi}
$$

Для нахождения в $(28)$ математического ожидания $\mathbf{E} S_{m}$ воспользуемся тем, что (см. (25)) $\mathbf{P}\left(|N| S_{m} \leqslant x\right)=\operatorname{th}(x / 2)$. Тогда найдем, что

$$
\mathbf{E}|N| S_{m}=\int_{0}^{\infty}\left(1-\operatorname{th} \frac{x}{2}\right) d x=\int_{0}^{\infty} \frac{e^{-x / 2} d x}{\operatorname{ch}(x / 2)}=4 \int_{0}^{1} \frac{y d y}{1+y^{2}}=2 \ln 2 \text {. }
$$


В силу независимости $N$ и $S_{m}$

$$
\mathbf{E}|N| S_{m}=\mathbf{E}|N| \mathbf{E} S_{m}=\sqrt{\frac{2}{\pi}} \mathbf{E} S_{m} .
$$

Значит,

$$
\mathbf{E} S_{m}=\sqrt{\frac{\pi}{2}} \mathbf{E}|N| S_{m}=\sqrt{\frac{\pi}{2}} 2 \ln 2=\sqrt{2 \pi} \ln 2,
$$

и, следовательно,

$$
\mathbf{E} \mathbb{D}_{1}=\mathbf{E} \sqrt{1-g} \mathbf{E} S_{m}=\frac{2}{\pi} \sqrt{2 \pi} \ln 2=\sqrt{\frac{8}{\pi}} \ln 2 .
$$

\section{СПИСОК ЛИТЕРАТУРЫ}

1. Borodin A.N., Salminen P. Handbook of Brownian Motion. Facts and Formulae. Basel: Birkhäuser, 1996. 462 p.

2. Carmona Ph., Petit F., Pitman J.W., Yor M. On the laws of homogeneous functionals of the Brownian bridge. Prepublication № 441. Paris: Laboratoire de Probabilités de l'Université Paris VI, Avril 1998.

3. Donati-Martin C., Shi Z., Yor M. A $[0,1]^{2}$-valued Brownian functional with arcsine marginals. Prepublication № 445. Paris: Laboratoire de Probabilités de l’Université Paris VI, Avril 1998.

4. Doob J. L. Heuristic approach to the Kolmogorov-Smirnov theorems. - Ann. Math. Statist., 1949, v. 20, p. 393-403.

5. Feller $W$. On the Kolmogorov-Smirnov limit theorems for empirical distributions. Ann. Math. Statist., 1948, v. 19, p. 177-189.

6. Feller $W$. The asymptotic distribution of the range of sums of independent random variables. - Ann. Math. Statist., 1951, v. 22, p. 427-432.

7. Kolmogoroff $A$. Sulla determinazione empirica di una legge di distribuzione. Giorn. Instit. Ital. Attuari, 1933, v. 4, p. 83-91.

8. Pitman J.W., Yor M. Quelques identités en loi pour les processus de Bessel. Astérisque, 1996, № 236, p. 249-276.

9. Revuz D., Yor M. Continuous Martingales and Brownian Motion. Berlin: SpringerVerlag, 1994, $560 \mathrm{p}$.

10. Ширяев А. Н. Основы стохастической финансовой математики. Т. 1, т. 2. М.: ФАЗИС, 1998. 512 c., 544 c.

11. Yor M. Some Aspects of Brownian Motion. Part I: Some Special Functionals. Part II: Some Recent Martingale Problems. Basel: Birkhäuser, 1992 (part I); 1997 (part II). 148 p.; 144 p. 